\title{
El tiempo libre educativo como recurso para la inclusión de las personas migrantes y refugiadas: diagnóstico y aproximación a las buenas prácticas en la Comunidad Autónoma Vasca
}

\author{
Gorka Roman Etxebarrieta
}

Departamento de Didáctica de la Lengua y la Literatura, Universidad del País Vasco/ Euskal Herriko Unibertsitatea (UPV-EHU)

gorka.roman@ehu.eus

\section{Israel Alonso Sáez}

Departamento de Didáctica y Organización Escolar, Universidad del País Vasco/Euskal Herriko Unibertsitatea (UPV-EHU)

israel.alonso@ehu.eus

\section{Naiara Berasategi Sancho}

Departamento de Didáctica y Organización Escolar, Universidad del País Vasco/Euskal Herriko Unibertsitatea (UPV-EHU)

naiara.berasategi@ehu.eus

Honako artikuluak Erasmus+ InclusiON izeneko proiektuan bildutako estrategia eta praktika onak aurkeztu nahi ditu. Bertan Europara heldutako etorkinen eta errefuxiatuen gizarteratzeko baldintzak eta jarrerak hobetzea bilatzen da. Erasmus+ InclusiON proiektu honen baitan Europako herrialde desberdinetako erakunde eta unibertsitateak daude, besteak beste, Eslovakia, Portugal, Italia eta Euskadi. Proiektuak aurrera egin ahala, Euskadin eta aurretik aipaturiko herrialdeetan zenbait bilera antolatuko dira. EAEn egindako azken bi bileren emaitzak aurkezten dira lan honetan, alde batetik eragozpenak eta aukerak izeneko atalean bildutakoak, eta bestetik momentuan martxan jartzen ari den zenbait praktika egoki. Azken hauen xedea, Euskadi eta Europara heldutako etorkin eta errefuxiatuen gizarteratzea, aisialdiaren bitartez bermatzea litzateke.

\section{GAKO-HITZAK:}

Gizarteratzea, aisialdia, immigrazioa, aisialdi hezitzailea, gizarte-politika.
El presente artículo tiene como objetivo presentar una serie de estrategias y buenas prácticas recogidas dentro del Proyecto Erasmus+ Inclusion, con la finalidad de facilitar y fomentar la inclusión de personas migrantes y refugiadas en Europa. En el proyecto Erasmus+ InclusiON participan varias entidades y universidades de Eslovaquia, Portugal, Italia y el País Vasco. A través del proyecto se prevé la realización de una serie de seminarios locales e internacionales; este artículo presenta algunas de las conclusiones recabadas en dos de las jornadas locales realizadas en el País Vasco. De entre estas últimas, se selecciona y presenta un relevante número de barreras y necesidades, así como una serie de buenas prácticas que se están llevando a cabo en el presente con el objetivo de fomentar la inclusión de personas inmigrantes y refugiadas a través del ocio y el tiempo libre.

\section{Palabras Clave:}

Inclusión, ocio, inmigración, tiempo libre educativo, política social. 


\section{Introducción}

La Unión Europea (UE) y sus regiones se enfrentarán en las próximas décadas a dos retos fundamentales e interrelacionados entre sí; el cambio demográfico en el seno de sus sociedades y la integración de las personas inmigrantes y las minorías étnicas. Como plantea Fertig (2010) a pesar de que la integración de este segmento de la población es un proceso que lleva varias décadas manifestándose en diferentes países de la UE, hoy por hoy no conocemos bien todavía cuáles son las estrategias y políticas necesarias para lograr la integración plena e inclusión de estos colectivos. Por ello, sigue siendo necesaria todavía una reflexión e investigación sobre esta cuestión, que arroje luz sobre una posible gestión y puesta en marcha de políticas de inclusión efectivas. En la literatura publicada hasta el momento, se puede apreciar que un aspecto clave en este proceso de integración es el ocio y la participación en actividades socioculturales.

En una amplia revisión de más de un centenar de investigaciones, Stoldoska (2015) concluye que el ocio juega un papel importante en la vida de los grupos étnicos y minorías raciales. Este ofrece una serie de beneficios, como la facilitación de contactos interculturales e intergrupales, oportunidades para el aprendizaje e intercambio cultural, fortalecimiento de los lazos con la comunidad, la preservación de su cultura, o aspectos como la importancia de la actividad física en el bienestar físico, mental y social de estas personas (Gonzalez-López et al., 2015).

Stodolska y Alexandris (2004) describen cómo los inmigrantes, especialmente en el período inmediato después de su llegada, a menudo utilizan el ocio y el deporte para restablecer su sentido de la valía, el valor personal dentro de la comunidad. En este sentido, el ocio también proporciona mecanismos para liberar y gestionar tensiones en un entorno estresante como es el que representa la llegada de un migrante a una sociedad desconocida (Hibbler y Shinew, 2002).

Cabe recordar que son múltiples los autores que aluden al hecho de que las personas migrantes que se desplazan a nuevos contextos socioculturales en busca de nuevas oportunidades sufren este tipo de procesos traumáticos derivados del choque cultural al que se enfrentan tanto ellos como el capital etnocultural con el que viajan (Berry et al., 2006; Lueck y Wilson, 2010). Y es que, en palabras tanto de Berry (1997) como de Gibson (2001), el abismo cultural que existe entre sociedad local e inmigrantes puede llegar a ser altamente estresante para los últimos.

La literatura también nos muestra la existencia de dificultades para el acceso a los recursos y entornos recreativos. Las actitudes racistas o de temor exhibidas por parte de la población local (Blahna y Black, 1993; Flood y McAvoy, 2007; Livengood y Stodolska, 2004; Sharaievska et al., 2010) así como la falta de información y conocimiento sobre recursos y oferta de ocio, o de las geografías de la población inmigrante en la ciudades, que en un gran porcentaje solo tiene la posibilidad de vivir en zonas degradadas y donde los entornos de ocio, como parques o espacios comunitarios, son percibidos como peligrosos y por lo tanto no son utilizados para el ocio de una manera normalizada (Stoldoska, 2015).

También podemos destacar que la investigaciones previas han planteado que las formas de entender el ocio son distintas según las diferentes procedencias culturales, así como entre las diversas generaciones de población inmigrante (Höglhammer et al., 2015). En una reciente investigación llevada a cabo en Alemania (Fertig, 2010) en la que se comparaban las actividades de ocio y las actitudes entre alemanes nativos y extranjeros inmigrantes de diferentes generaciones, se apreciaba que las actitudes de los inmigrantes de segunda generación tienden a caracterizarse por un mayor grado de fatalismo y pesimismo sobre los demás grupos, aunque sus actividades de ocio y la participación en la vida social se asemejen más a las de los alemanes nativos que en la generación de sus padres.

Existen, por otro lado, estudios que evidenciarían la correlación existente entre el grado de participación en las actividades de ocio y la generación a la que el sujeto migrante pertenecería. En este sentido, encontramos estudios como el llevado a cabo por Liu et al. (2009) en Estados Unidos, donde se afirma que la relación existente entre las actividades de ocio y el deporte, por una parte, y los procesos de aculturación, por otra, sería más obvia y efectiva entre los adolescentes hispanos de segunda y tercera generación. No obstante, estudios como el de Stodolska y Alexandris (2004) constatan que las actividades de ocio fomentarían la aparición de oportunidades para los inmigrantes de primera generación tanto a la hora de participar de la arena de lo social como a la de afianzar sus lazos etnoculturales.

\subsection{Tiempo libre educativo e inclusión}

Hoy en día es indiscutible el papel esencial que desempeña el ocio en el desarrollo personal y social en el seno de las sociedades modernas. Diferentes estudios, plataformas y foros declaran que el ocio es un espacio que posibilita la creatividad, la relajación y la autorrealización (Höglhammer et al., 2015) convirtiéndose en un elemento que no se puede dejar de lado en los asuntos relacionados con la política cultural y social. Pero no solo eso, sino que el ocio y la acción sociocultural también se pueden entender como un elemento clave la hora de promover la inclusión y las comunidades inclusivas. Estaríamos hablando de la inclusión como efecto del ocio y tiempo libre educativo (TLE), pero también como perspectiva desde la que se plantea que estas actividades sean inclusivas, es decir, que acepten la diversidad y la diferencia como 
valor, y que todas las actividades que se realicen posibiliten la participación de todas las personas sin discriminación de ningún tipo (Madariaga, 2004). Se trataría de que todas las personas puedan ser valoradas y tener voz partiendo de las capacidades que cada uno o una tiene. Estamos, por lo tanto, hablando de actividades que fomenten la participación activa y la comunicación de colectivos, como es el caso de los que presentan diversidad funcional, ya que normalmente estos ven limitada esa participación (Fantova, 2004).

Ainscow (2004), referente en este campo, realiza un análisis de la literatura relacionada con la educación inclusiva y cita cuatro pilares de esta que podrían ser extrapolados a otros contextos, como el de las iniciativas de ocio y tiempo libre educativo. Estos serían los siguientes:

1. La inclusión como proceso. Es decir, la inclusión ha de ser vista como una búsqueda de mejora constante para responder a la diversidad de las personas participantes. El objetivo es aprender a vivir con la diferencia y a la vez de estudiar cómo podemos sacar partido a esta. En este sentido, las diferencias son percibidas como un estímulo para fomentar el aprendizaje entre diferentes en las actividades de tiempo libre.

2. La inclusión debe buscar la presencia, la participación y el éxito de todas las personas. El término participación se refiere a la calidad de sus experiencias, a incorporar sus voces y a la valoración de su bienestar personal y social.

3. La inclusión precisa de la identificación y la eliminación de barreras que impidan el ejercicio efectivo de los derechos, en este caso, un ocio inclusivo.

4. La inclusión pone particular énfasis en aquellos grupos de personas que podrían estar en riesgo de marginalización, exclusión o fracaso escolar.

Como comentábamos anteriormente, un aspecto básico de la inclusión es la aceptación de la diferencia (Devine y Dattillo, 2000), especialmente en el caso de las personas que provienen de procesos migratorios. Estos podrían además tener necesidades e intereses diferentes a las personas autóctonas que normalmente diseñan este tipo de actividades. Por ello, es imprescindible que las personas promotoras conozcan, entiendan y valoren esa diversidad social y cultural. Otro tipo de acercamiento entendería la inclusión como un derecho, como un planteamiento filosófico y político que afirmaría que todas las personas deben ser respetadas para poder participar de igual manera en el seno de la sociedad (MacNeil y Anderson, 1999). Teniendo en cuenta estos aspectos, todo proyecto sociocultural de tipo inclusivo debería respetar los siguientes principios (Morata et al., 2015):

- Toma como referente el ideal democrático de la sociedad.

- Incluye a todos los miembros de la comunidad.
- Es una filosofía institucional que implica actitudes, valores y creencias.

- Desarrolla el sentido de comunidad, de apoyo y de trabajo en equipo.

- Mejora la calidad y relevancia de las oportunidades y experiencias.

- Considera a todas las personas protagonistas en primera persona de las actividades de ocio, teniendo en cuenta sus características, necesidades e intereses.

- El interés se centra en prestar apoyo a las necesidades de cada persona.

- Se ofrece el apoyo en contextos naturales y grupos heterogéneos.

- Se trabaja desde una perspectiva institucional.

- Las dificultades se afrontan como una oportunidad para mejorar el sistema.

- La entidad y/o el equipamiento se transforma para responder a los usuarios.

- La atención se centra en los recursos ofrecidos para responder a todos.

Para estos autores, la inclusión en un planteamiento de TLE está definida por los siguientes elementos: a) se considera un derecho humano; b) se dirige a todas las personas; c) se basa en un modelo sociológico; d) se apuesta por la inclusión total y global de toda persona en cualquier ámbito; e) implica una reforma estructural; f) se toma como referencia la comunidad y el entorno social; g) plantea una oferta común para todas las personas, se articula desde una organización inclusiva; y h) supone un compromiso en tanto en cuanto se debe adaptar la realidad a las necesidades de cada persona y adecuar la oferta a usuarios distintos por diferentes razones.

Stoldoska (2015) plantea que una oferta de ocio sociocultural para los miembros de comunidades y minorías étnicas debería considerar: a) conocer y partir de sus necesidades, para lo que es importante acceder a la información mediante diferentes técnicas y estrategias (encuestas, grupos focales, entrevistas, etc.); b) flexibilidad y apertura en la oferta; c) pensar en las barreras existentes a las que hay que hacer frente e implementar la participación activa de todas las personas; d) ser conscientes de la historia de la comunidad en cuestión y observar posibles tensiones interculturales o interétnicas que podrían haber existido en el pasado; e) ser conscientes de que algunos inmigrantes pueden viajar con historias y recuerdos relacionados con sus experiencias como refugiados o de conflictos étnicos pretéritos que experimentaron en el pasado y que podrían afectar a su confianza a la hora de relacionarse con otros grupos étnicos; f) desarrollar y establecer colaboraciones y redes sociales con otros grupos y asociaciones de la comunidad; y e) tener en cuenta que el ocio y las actividades socioculturales pueden ser utilizadas de forma creativa para analizar barreras, construir puentes entre los diferentes miembros de la comunidad y ayudar a que los recién llegados se adapten al nuevo entorno a través de actividades que involucren un contacto intergrupal e intercambio entre culturas y aprendizajes. 
Ayarza et al. (2010) plantean que una de esas iniciativas de ocio son los grupos de tiempo libre educativo, subrayando la importancia de su función como constructores de espacios educativos, de humanización y de transformación social, ya que estos permiten, a través de la participación y la reflexión crítica, dotar a las personas de valores de convivencia, de sociabilidad y de implicación activa en asuntos colectivos. Estos promueven, además, la solidaridad, el respeto al entorno, la iniciativa personal y social o el cuidado e inclusión del resto de personas. Armengol (2010) plantea que los organizadores de actividades de tiempo libre son muy diversos. En este sentido, al movimiento de tiempo libre citado habría que añadir las diversas actividades promovidas desde las Administraciones Públicas, empresas privadas, entidades de servicios, clubes deportivos ${ }^{1} \mathrm{y}$ otros estamentos.

Recientes estudios muestran que la población inmigrante en el Estado español ha tenido un gran crecimiento. En los últimos veinte años, España se ha convertido en el país que más personas inmigrantes ha acogido de todo el continente europeo (Malmusi, Jansa y Del Vallado, 2007). En la primera década del siglo XXI, la población inmigrante en España se incrementó de un 1,8\% a un $11,4 \%$. En la actualidad el porcentaje de personas inmigrantes se situaría en un 12,2\% (INE, 2016). No obstante, este porcentaje variaría entre comunidades autónomas. En el caso del País Vasco, y según los datos aportados por Ikuspegi-Observatorio Vasco de Inmigración ${ }^{2}$, la población migrante habría pasado de 26.334 personas en 1998 a 195.969 en 2017 , lo que supondría un incremento del $744 \%$ en poco menos de una década. El grupo más importante en cuanto a su procedencia es el que conforman los migrantes latinoamericanos, con un total de 91.510 personas, seguido por los que proceden del Magreb, con un total de 27.441 personas, $y$ las personas de origen subsahariano, que serían 15.182 en total.

Si nos fijamos en los términos porcentuales correspondientes a su presencia en el seno de la sociedad local, veremos que la población migrante representa el 8,9\% de la población total en la Comunidad Autónoma Vasca (CAV). Es por esta razón que debemos afrontar con rigor y seriedad institucional la gestión y promoción de la inclusión de las personas migrantes y refugiadas en el País Vasco, ya que son parte activa de nuestra sociedad y representan a una parte importante de esta.

\section{Metodología}

El presente artículo debe contextualizarse dentro del proyecto Europeo Erasmus+ InclusiON³, en el que participan seis organizaciones colaboradoras de cuatro países; Italia, Portugal, Eslovaquia y País Vasco (tres de ellas, Kiribil, EDE Fundazioa y la Universidad del País Vasco/Euskal Herriko Unibertsitatea -UPV/EHU-, serían organismos ubicados en el País Vasco). Este proyecto tiene como objetivo la investigación, recolección e intercambio de posibles buenas prácticas y de metodologías del tiempo libre educativo orientadas a proyectos de inclusión social, así como al desarrollo de programas y acciones para afianzar el TLE como herramienta de inclusión social de las personas inmigrantes. El proyecto se está llevando a cabo desde enero de 2017 y concluirá en junio de 2018. Para realizar el proyecto se prevén una serie de seminarios locales e internacionales (véase el Gráfico 1), de los cuales algunos ya han completados (Bilbao, Bratislava y Nápoles).

Gráfico 1. Cronograma de seminarios locales y europeos del proyecto Erasmus+ InclusioN

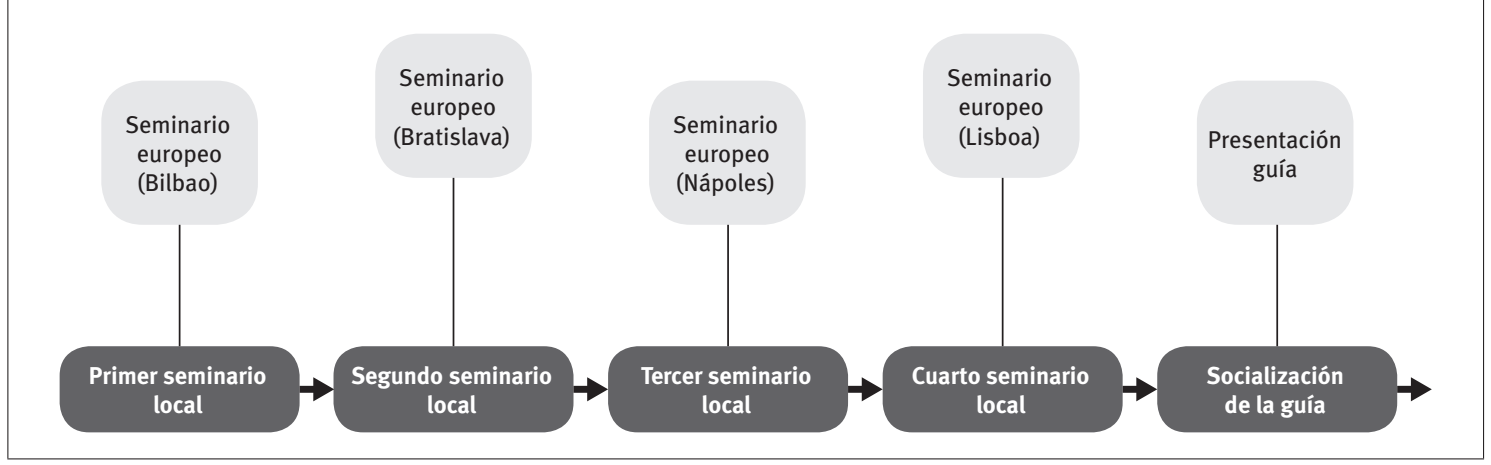

Fuente: Elaboración propia.

${ }^{1}$ Para Ayarza et al. (2010), las actividades de tiempo libre pueden surgir desde instancias con finalidades muy diversas y con objetivos y programas muy distintos. Nos podemos encontrar con ofertas de colonias, colonias urbanas, campamentos, centros de tiempo libre, etc., que tienen poco en común tanto desde el punto de vista del enfoque como de los contenidos específicos de la actividad. Al mismo tiempo, una actividad con idénticas u homólogas características puede recibir denominaciones distintas.

${ }^{2}$ Recuperado de [〈http://www.ikuspegi.eus/es.〉]. 
El presente artículo se construye a partir de los dos primeros seminarios locales realizados tanto en el País Vasco como en el resto de países. Los objetivos de estos seminarios y del artículo serían los siguientes:

- Detectar las buenas prácticas de TLE e inclusión con personas inmigrantes y refugiadas existentes en cada país.

- Detectar las oportunidades, barreras y necesidades para la inclusión en el tiempo libre educativo.

En la jornada llevada a cabo en el País Vasco durante mayo de 2017, se emplearon una serie de técnicas y procedimientos que se describen a continuación. En ella participaron treinta personas provenientes de diferentes sectores y entidades relacionadas con el objeto del proyecto (véase la Tabla 1). La mayoría de los asistentes estaban familiarizados o eran expertos en cuestiones relacionadas con el TLE, aunque también participó un número importante de personas inmigrantes pertenecientes a entidades sociales, además de los educadores y responsables de estas.

Las técnicas utilizadas en la sesión para el análisis de la situación y búsqueda de buenas prácticas fueron las siguientes:

a. Línea del tiempo. Cada persona debía explicar momentos en los que habría visto (por motivos personales o profesionales) la importancia del tiempo libre educativo en la inclusión de personas inmigrantes y refugiados, así como explicar por qué. La información se recogió directamente en un papelógrafo y posteriormente surgió un debate en torno a las ideas recopiladas.

b. World-café. Se debatieron en torno a una mesa las siguientes cuestiones: ¿qué necesidades y retos se aprecian a través del tiempo libre educativo con relación a la inclusión de las personas inmigrantes y refugiadas?; ¿qué formación sería necesaria para ello?

c. Barreras y oportunidades en relación con la inclusión de personas inmigrantes y refugiadas a través del ocio y el tiempo libre. Se instalaron dos grandes papelógrafos, bajo los títulos de "Barreras" y "Oportunidades", para que cada uno de los asistentes pudiese plasmar en ellos las posibles barreras y oportunidades que apreciaran en los procesos de inclusión de personas inmigrantes y refugiadas, con el fin de debatirlos después en un coloquio abierto entre todos los participantes.

d. Búsqueda de buenas prácticas en relación con la inclusión a través del tiempo libre educativo. Cada persona realizó un dibujo relacionado con alguna buena práctica que hubiese vivido o conociese, relacionada con la temática del proyecto. Además del dibujo, cada persona realizó una pequeña redacción para explicar dónde se había llevado a cabo y de qué trataba. Posteriormente todo ello se presentó y discutió en un debate en el que todos y todas los y las asistentes tomaron parte.

Tabla 1. Sexo y entidad de los participantes

\begin{tabular}{|c|l|}
\hline Sexo & Entidad \\
\hline H & Trabajador de Kiribil Sarea \\
\hline H & Monitor en Aisibizia Elkartea \\
\hline M & Educadora en Harribide Fundazioa \\
\hline M & Trabajador en Alboan \\
\hline M & Trabajadora de Bilboko Udala \\
\hline H & Trabajador de cooperativa \\
\hline M & Trabajadora en Bizkaiko Urtxintxa Eskola \\
\hline M & Trabajadora en Gaztetxo Eskola \\
\hline M & Miembro de Gaztetxoen Koordinakundea \\
\hline M & Participante en Mujeres con Voz \\
\hline M & Monitora en Amesten Elkartea \\
\hline M & Monitora en Amesten Elkartea \\
\hline M & Aurrerantz, plataforma de voluntariado \\
\hline H & Aisibizia Elkartea \\
\hline M & Gozaldi gazte elkartea \\
\hline H & Monitor de Argizai Eskaut Taldea \\
\hline M & Profesora de Escuela de Tiempo Libre EDE \\
\hline
\end{tabular}

\begin{tabular}{|c|l|}
\hline Sexo & Entidad \\
\hline M & Trabajadora de Gaztegune Uribarri \\
\hline M & Profesional de EDE Eskola \\
\hline H & Alumno de Educación Social \\
\hline H & Alumno del grado de Educación Social \\
\hline H & Miembro de Euskalerriko Eskautak Bizkaia \\
\hline M & Monitora de Argizai Eskaut Taldea \\
\hline H & Dinamizadora en Ainara kultur Elkartea \\
\hline M & Educadora en Giltzarri (Ayuntamiento de Barakaldo) \\
\hline M & Argizai Eskaut Taldea \\
\hline H & Participante en Fundación Harribide \\
\hline H & Participante en Fundacion Harribide \\
\hline H & Suspergintza elkartea \\
\hline H & Trabajador en Biltzen \\
\hline H & Educador en Izangai Elkartea \\
\hline H & Participante en Izangai Elkartea \\
\hline H & Monitor en Eskubeltz Eskaut Taldea \\
\hline
\end{tabular}

Fuente: Elaboración propia. 
Las treinta personas que participaron en el seminario local de Bilbao se organizaron en cuatro grupos. Se asignó además a cada uno de los grupos un dinamizador y un relator para que dinamizaran el trabajo y la comunicación del grupo y recogiesen por escrito toda la información aportada por cada uno de los componentes. Cada grupo realizó una tarea independiente de las del resto de grupos. Posteriormente, estas tareas se rotaron a los otros grupos para que se continuase trabajando a partir de lo que anteriormente ya había realizado el resto de participantes.

Toda la información obtenida de las tareas y debates internos de cada grupo fue grabada y posteriormente transcrita. Con esta información y con la aportación escrita de cada uno de los relatores, se realizó un posterior análisis a partir de una serie de categorías que ha permitido a posteriori inferir los resultados que desarrollamos en el siguiente apartado.

\section{Resultados}

\subsection{Necesidades y retos del tiempo libre educativo para la inclusión de las personas inmigrantes y refugiadas}

Un primer grupo de necesidades y retos está relacionado con el desconocimiento que se tiene acerca de la realidad de las personas que provienen de procesos migratorios y de refugio, y que se da en el seno los grupos de TLE y los dispositivos socioculturales. Se señala la importancia de estar y trabajar con ellos y ellas, para así poder generar espacios de diálogo desde los que construir estrategias y plantear actuaciones que respondan a sus necesidades. Se considera asimismo imprescindible conocer las posturas, puntos de vista y cosmologías de estos con la intención de saber qué es lo que quieren, qué les gusta o qué necesitan. Presentamos a continuación algunas de las cuestiones que se han planteado con relación a esta necesidad:

- Dar voz y espacios a los migrantes y refugiados para que se puedan expresar, ya que habitualmente se imponen y se dan por supuesto necesidades y opciones que estas personas no han expresado.

- Crear espacios de encuentro entre personas migrantes y locales. Esta no debería ser una actividad ocasional, ya que lo que se buscaría sería crear vínculos permanentes que se manifiesten en la vida diaria. Se trataría de normalizar y naturalizar esos encuentros en los que participen personas de diferentes edades.

En definitiva, se trataría de consolidar una apertura necesaria por parte de cada entidad o recurso de TLE hacia el contexto en el que se inserten, haciendo un análisis de la realidad del entorno (barrio, pueblo, etc.) de la entidad y de las personas y culturas que formen parte de esa realidad, en concreto de la situación de las personas que provengan de procesos migratorios o refugio.
En este proceso de generación de espacios comunes, se plantea también ir un paso más allá. No se trata simplemente de acercarse y escuchar, sino que ha de posibilitarse una participación activa de todos los actores. Se subraya además la importancia de que estos sean incorporados como sujetos activos con capacidad de generar, crear y decidir. Mostramos a continuación algunas de las necesidades y retos identificados:

- Es necesario abrir y socializar los recursos y los espacios de tiempo libre para que las personas migrantes y refugiadas los conozcan y tomen parte en ellos.

- Sería deseable que las personas emigrantes organicen y formen parte de los recursos de tiempo libre. De esta manera, no solo serían parte activa al conseguir objetivos asociados a la inclusión, sino que se lograría, gracias a las redes sociales de estos participantes, animar a más personas migrantes y refugiadas a participar de este tipo de recursos.

- En esta misma línea, cabe señalar que se detecta en jóvenes migrantes una motivación intrínseca de superación y participación en los recursos socioculturales y de tiempo libre.

Para realizar este camino de acercamiento y generación de puentes de conocimiento y comunicación con las personas que provienen de procesos migratorios, así como para promocionar su participación en este tipo de actividades, se han identificado algunas actitudes y planteamientos de fondo que deberían tenerse en cuenta por parte de los responsables de estas iniciativas:

- No tratar a los inmigrantes como un único colectivo, ya que dependiendo de su procedencia, idioma, modo de llegada o necesidades, vivencias e inquietudes, pueden no percibir su pertenencia a un grupo en concreto.

- Desterrar y romper con prejuicios que se experimentan de un modo más marcado en adultos, ya que se observa que los niños y los jóvenes son más abiertos y tolerantes con la diversidad etnocultural.

- Tener en cuenta que a menudo el rechazo se fundamenta en el nivel socioeconómico, más que en la procedencia de la persona migrante.

- Evitar que las segundas y terceras generaciones (y tampoco las primeras generaciones) se sientan como personas extranjeras o inmigrantes.

- Gestionar la diversidad cultural desde el respeto y la inclusión de todas y cada una de las personas.

- Romper con los estereotipos y falsos rumores sobre esta población, algo que resulta imprescindible.

Otro grupo importante de necesidades y retos detectado estaría relacionado con la adecuación de la metodología y las formas de hacer en las actuaciones 
o grupos de TLE y equipamientos socioculturales. En concreto se realizan las siguientes indicaciones:

- Se considera necesario acabar con las actuaciones e intervenciones desde el paternalismo. En la medida de lo posible debemos normalizar y no distinguir por colectivos, así como evitar llevar a cabo discriminaciones de tipo negativo/positivo.

- Es recomendable la creación de grupos y alternativas centradas en las necesidades de las personas migrantes que mantengan un equilibrio tanto con sus necesidades como con los objetivos que se quieren conseguir. Asimismo, se deberían llevar a cabo proyectos cercanos y accesibles para que la gente se acerque por voluntad propia y no haya que acudir en busca de personas dispuestas a participar.

- Se debe adaptar no solo el colectivo a la sociedad de acogida, como se intenta hacer, sino que el proceso debe ser bidireccional y poder así adaptar también el entorno y la sociedad de acogida a las personas migrantes. De este modo, el proceso de inclusión se haría mediante la implicación de las dos partes y no solo por parte de las personas inmigrantes.

- Se debería evitar la creación exclusiva de dinámicas o actividades específicas para la inclusión, con el fin de posibilitar que todas las dinámicas sean inclusivas e inclusoras. Debería evitarse a su vez que la educación en la interculturalidad y en la inclusión de personas inmigrantes se quede en actividades y momentos ocasionales y no se trate de manera trasversal.

- Resulta necesario incorporar a las familias inmigrantes de una misma procedencia en espacios de tiempo libre para reafirmar y asentar la identidad cultural de sus hijas e hijos.

- Sería también recomendable contar las experiencias personales de jóvenes inmigrantes a la hora de romper con los estereotipos, pero a la vez no caer en la sistematización del testimonio como única herramienta de trabajo e inclusión en el ámbito intercultural. Es importante no caer en la pena para poder trabajar la sensibilización sobre el colectivo de personas inmigrantes.

- Al realizar las actividades propuestas, se debería tener en cuenta que los jóvenes inmigrantes no tienen a menudo acceso a los recursos económicos con los que la mayoría de los jóvenes "autóctonos" cuentan para acceder a la oferta de ocio.

- Se han de distinguir las diferentes etapas del desarrollo en el tiempo libre, teniendo en cuenta que no siempre lo que se hace a unas edades es efectivo en otras.

- Por último, resulta imprescindible plantear un tiempo libre educativo no solo para la infancia y juventud temprana, sino hacerse también con personas adultas, ya que en su mayoría los recursos de tiempo libre están enfocados en niñas y niños, y por tanto las personas adultas se quedan a menudo sin este tipo de alternativas.

Otra necesidad detectada es la de difundir y dar a conocer el trabajo que se está realizando en torno al TLE. En otras palabras, resulta necesario que las personas inmigrantes y el resto de la comunidad conozcan las iniciativas que se están llevando a cabo y el potencial que estas tienen. En este sentido, se plantea como reto estar a pie de calle y acercarse a los barrios para poder conocer y atraer a las personas a los recursos de tiempo libre y a las diversas asociaciones. También resulta imprescindible la creación de redes de comunicación que den a conocer los recursos en los que se puede participar.

Existe otro grupo de necesidades y retos que estaría relacionado precisamente con el trabajo en red. Los diversos grupos e iniciativas de tiempo libre ven necesario trabajar y converger con otras iniciativas, agentes y dinámicas sociales de otros ámbitos, y poder así cumplir los siguientes objetivos:

- Recuperar la calle y lo comunitario. Dar accesibilidad a las actividades y dinámicas de la calle para que la gente pueda participar de manera espontánea sin tener que pasar por los trámites burocráticos que distancian a la población de la participación.

- Crear redes entre profesionales, recursos, grupos y personas. Potenciar un trabajo en red no solo de asociaciones específicas de este ámbito, sino también de colaboración entre diferentes grupos, instituciones, profesionales, etc.

- Como agentes sociales, tomar conciencia y asumir un papel activo en la acción política.

- Crear un mapa de recursos, entidades, grupos, profesionales y buenas prácticas, acompañado de una guía que recoja las buenas prácticas más destacadas, con el fin de hacer del mapa un recurso práctico no saturado. Esta guía no solo sería utilizada por profesionales, sino que también tendría que ser útil para personas inmigrantes. Además de esta guía, se plantea la necesidad de un espacio donde se tenga acceso a información relevante, como documentos de referencia que puedan ser utilizados. En esta guía deberían incluirse las mesas que tiene el Gobierno (mesa de juventud, de la mujer, de inmigración, etc.) así como los recursos que estas ofrecen.

- Integrar y fomentar la participación de las familias.

- Potenciar y hacer funcionar programas antirrumores en todos los ayuntamientos. Trabajar con los diversos medios de comunicación para que estos no informen creando falsos rumores y fortalecer así estereotipos que dificulten la convivencia.

- Realizar un análisis de la realidad de las ciudades, pueblos y barrios para poder conocer las buenas prácticas de otras entidades, grupos e 
instituciones tanto dentro como fuera de nuestro territorio.

También se destaca la necesidad de cobertura y apoyo institucional para el buen funcionamiento de las iniciativas anteriormente propuestas. En concreto se destacan las necesidades de:

- Colaboración política (junto a las asociaciones y grupos de tiempo libre y scout implicadas en dinámicas y programas de TLE) en la cobertura de las necesidades básicas de las familias y del resto de participantes. Se observa que desde las asociaciones y diversos grupos no solo se ofrece un espacio de ocio y educación si no que se intentan satisfacer otras necesidades básicas como la de manutención durante breves periodos de tiempo (campamentos, colonias, actividades de día, etc.). Los grupos mencionados no pueden hacer frente a esta problemática por si solos, por lo que es necesario solicitar colaboración a las instituciones. En este sentido se demanda a las diversas instituciones que ofrezcan:

- Facilidades, sobre todo por parte de los Ayuntamientos, para ocupar las calles y poder

Tabla 2. Barreras y oportunidades detectadas para la inclusión de las personas inmigrantes y refugiadas

BARRERAS
Relacionadas con las personas que provienen de procesos
migratorios
- Lenguaje e idioma.
- Mentalidad y cultura (ritmos, horarios, etc.).
- Algunas expresiones religiosas.
- Falta de compromidad es la familia.
- En muchos casos tienen otras necesidades/prioridades previas
como el trabajo o la vivienda.
Relacionadas con las actividades y las iniciativas llevadas a cabo
- Las propuestas llevadas a cabo no siempre están adaptadas a sus
necesidades. Se desarrollan iniciativas y propuestas sin haber
consultado a las personas inmigrantes.
- Posible desinformación o desconocimiento acerca de los
espacios/actividades de ocio y tiempo libre educativo.
mo se sale en la búsqueda de las personas migrantes, sino que a
voluntad.

Relacionadas con actitudes de las personas que llevan a cabo las iniciativas 0 actividades de TLE

- Cuando se piensa en la inclusión de personas migrantes, nos limitamos a la cultura y el folclore por qué se desconoce su verdadera realidad.

- Existen problemas a la hora de involucrarse de un modo natural.

- Se manifiesta la existencia de "guetos blancos".

- Existe una imagen estereotipada que se traduce en una mitificación de la problemática.

- Existen una serie de prejuicios, como puede ser el origen de las personas migrantes.

- Se ve a las personas migrantes como un problema y no como una oportunidad.

- Existen multitud de rumores que dificultan todo el proceso.

- Los medios de comunicación a menudo actúan como altavoces de esta rumorología aumentando la alarma social.

- Existe un desconocimiento mutuo entre la población local y los migrantes.

- El recelo se da de un modo bidireccional.

\section{Relacionadas con recursos}

- Hay escasos recursos económicos destinados a las actividades y programas de tiempo libre educativo.

- Existe una falta de formación para formar a las personas encargadas de acompañar a los migrantes.

- No existe un mapa o guía que recoja las distintas iniciativas de tiempo libre educativo.

- Se percibe una falta de coordinación con otras asociaciones y agentes del entorno.

OPORTUNIDADES

Relacionadas con las personas que provienen de procesos migratorios

- Interés para participación en actividades de TLE una vez resueltos los problemas.

- Sentimiento comunitario más extenso.

- La diversidad y juventud que caracteriza a este grupo.

- Ganas de compartir.

- Contar con la segunda generación.

- Existencia de actividades que reflejan un modo de vida, de cocinar, de disfrutar del ocio, etc. que está abierta a la comunidad y que puede así enriquecerla.

- Riqueza gastronómica en una tierra donde este sector se encuentra en un proceso de continuo fortalecimiento.

Impacto de estas iniciativas

- La infancia no parte con los prejuicios de la población adulta por lo que pueden vivir estos procesos de forma natural.

- Se da la oportunidad de conocer otras personas.

- Existe un enriquecimiento personal y cultural.

- Se favorecer la interacción entre el colectivo de inmigrantes y los locales.

- Se implementan las relaciones igualitarias de carácter horizontal, además de reconocer y potenciar lo que nos une y no lo que nos separa.

- Surgen nuevas experiencias y realidades.

- Se crean vasos comunicantes que abren otras puertas.

- En el ámbito de la sensibilización se da un cambio en las mentalidades y cosmovisiones de las personas.

\section{La diversidad como valor}

- La diversidad se presenta como un elemento enriquecedor y valor añadido y no como un elemento perturbador para la inclusión

social.

- Impulso de a la diversidad como riqueza cultural.

\section{El ámbito del TLE como oportunidad}

- Necesidad de reconocer el espacio del tiempo libre educativo como espacio de inclusión.

- Implementación de los canales de información compartida, así como del trabajo en red.

- Trabajo constante de un grupo de agentes de TLE caracterizados por la inquietud, la búsqueda de conocimientos y mejora continua.

- Participación de organizaciones y asociaciones de personas inmigrantes que demuestran sensibilidad por el desarrollo socio-educativo.

- Trabajo a nivel local en el barrio para facilitar la relación entre personas y grupos.

- Los grupos de tiempo libre educan mediante actividades y metodologías activas que resultan atractivas y tienen la posibilidad de "enganchar".

- Existe un gran número de buenas prácticas que se llevan a cabo a diario con la población migrante por parte de trabajadores del sector, servicios o asociaciones. 
llevar a cabo actividades fuera de los locales destinados a la realización de actividades de TLE.

- Una buena ubicación de los recursos ya que muchos de los que están destinados a personas inmigrantes se encuentran en el extrarradio de las ciudades y por tanto se contribuye a la exclusión social de estas personas mediante otra exclusión espacial.

Una última cuestión específica a la que el proyecto Erasmus+ InclusiON quiere responder, es la referente a la necesidad de conocer cuáles son las demandas formativas en este ámbito. Hemos dividido estas en dos grupos. El primero estaría relacionado con la formación necesaria para los responsables y educadores/monitores:

- Formación en temas de interculturalidad impartida por personas de diferente procedencia.

- Formación en un tipo de tiempo libre que prime el punto de vista de la inmigración, que atraiga a la población local.

- Formación para una correcta gestión e implementación del trabajo en red.

- Formación en materia de incidencia política para las personas profesionales del tiempo libre, para que no solo lleven a cabo actuaciones desde la calle, sino que también se llegue a dimensiones y decisiones desde la política.

- Formación en estrategias y recursos para trabajar la interculturalidad, la diversidad y la normalización de la convivencia con personas de diferente procedencia desde una edad temprana.

El segundo grupo está integrado por necesidades formativas no solo para estos monitores, 0 responsables de las actividades, sino también para las familias, otros agentes educativos o toda la comunidad. Se destaca la necesidad de una formación relacionada con la asimilación crítica de lo trasmitido en los medios de comunicación para evitar la creación de rumores, estereotipos y miedos. Es necesario, aludiendo de nuevo al programa "anti-rumores" 4 , subrayar la importancia de una comunicación sana, asertiva y libre de rumores y prejuicios. Por último, debemos incidir en la importancia de fomentar una educación orientada a la gestión y asimilación de la información que se publica en las redes sociales.

Existen dos últimas cuestiones a destacar en este apartado; por un lado la necesidad de formación e información a las familias acerca de los grupos de tiempo libre y que es lo que se hace en ellos, y por otro, el reconocimiento de la formación en TLE que las personas inmigrantes han recibido en sus países de origen.

${ }^{4}$ Consultar sitio web del proyecto: [〈http://antirumores.com〉]

\subsection{Barreras y oportunidades}

En la Tabla 2 se recogen los resultados relacionados con las barreras y oportunidades en la inclusión de personas inmigrantes y refugiadas a través del Ocio y Tiempo Libre en nuestro territorio.

\subsection{Una aproximación a buenas prácticas en nuestro entorno}

A continuación se destacan algunas de las buenas prácticas recogidas en la sesión de trabajo.

Posteriormente se realizará una aproximación a estas a través de entrevistas directas para verificar si efectivamente se trata de buenas prácticas teniendo en cuenta los criterios de innovación, sostenibilidad, inclusión e impacto, para poder así recoger toda la información sobre ella. Estas prácticas, junto a las obtenidas en Eslovaquia, Portugal e Italia formarán parte de una guía metodológica que se publicará en el 2018 (Tabla 3).

Tabla 3. Buenas prácticas para la inclusión de personas inmigrantes y refugiadas a través del ocio y tiempo libre

\begin{tabular}{|l|l|}
\hline Nombre del proyecto & Municipio \\
\hline Somos Rontegi Gara & Barakaldo \\
\hline Bizilagunak & Barakaldo \\
\hline Berbalagun: & Varios \\
\hline Bizilagunak: & Galdakao \\
\hline Munduko Arrozak & $\begin{array}{l}\text { Barrio San Francisco (Bilbao) y } \\
\text { otras localidades }\end{array}$ \\
\hline Giltzarri & Barakaldo \\
\hline Auzoko & $\begin{array}{l}\text { Diferentes pueblos de Gipuzkoa } \\
\text { (Hernani, Aixola, Bergara, }\end{array}$ \\
\hline Arrasate, Donostia, etc.)
\end{tabular}

\section{Conclusiones}

Uno de los retos a los que tanto la UE y como nuestras comunidades locales han de hacer frente, es la problemática derivada de los procesos de inclusión que implican a personas que provienen de procesos migratorios. Su notable crecimiento a lo largo y ancho del territorio durante las últimas décadas da una idea de la extensión del fenómeno migratorio en tierras vascas. El gran número de personas que siguen llegando para formar parte de nuestras sociedades, huyendo de conflictos políticos/armados o de la pobreza, será clave para el desarrollo social y cultural del futuro. Este hecho hace que resulte 
necesario plantearse que sistemas y espacios podrán garantizar su inclusión a corto, medio y largo plazo. Resulta en este sentido imprescindible llevar a cabo un trabajo de agentes similar al del sistema escolar o los servicios sociales, aunque también sería aconsejable recalcar la importancia que tiene la comunidad en su conjunto, así como las diferentes experiencias y actividades que cada persona inmigrante pueda desarrollar en todos los ámbitos y momentos, para conseguir que esté proceso de inclusión pueda llegar a buen puerto.

En esta misma línea sería correcto considerar que un espacio privilegiado para el fomento de esta inclusión que complementa y colabora con las desarrolladas en otros ámbitos, es la denominada Educación en el Tiempo Libre. Se trataría de espacios y recursos en los que se aprenden y adquieren valores cívicos y ciudadanos, disfrutando y experimentando a partir de sus intereses. Centrándonos en la infancia y la adolescencia, deberíamos mencionar la existencia de movimientos tradicionales como el escultismo o los grupos de Tiempo libre, pero también de recursos como las bibliotecas, ludotecas, gaztegunes, colonias urbanas, campamentos, actividades comunitarias, procesos participativos con niños y jóvenes, programas de educación de calle, etc. que tendrían una finalidad educativa de desarrollo y adquisición de valores humanos y sociales y transformación social.

En las últimas décadas se ha ido desarrollando en el País Vasco un sistema de recursos en este ámbito. Este ha sido en ocasiones impulsado por agentes sociales, aunque en otras lo ha sido por las diversas instituciones públicas. En el presente un gran número de los niños, niñas y adolescentes de nuestra comunidad participan activamente en varios de estos espacios. Del mismo modo que hemos planteado a lo largo de este trabajo, todos los espacios mencionados han de ser inclusivos, para poder así convertirse en elementos activadores y posibilitadores de la inclusión de estas personas. En este sentido diferentes investigaciones apuntan a que, estos espacios están posibilitando la integración y el encuentro. Estos además estarían abiertos a esa realidad sociocultural anteriormente mencionada, posibilitando la construcción a su alrededor de diversas buenas prácticas que podrían servir como referencia para seguir avanzando en esta dirección. Por otro lado se pone de manifiesto que aún quedaría mucho camino por recorrer. Resulta necesario por lo tanto que estos dispositivos no solo sean flexibles y se adapten a las necesidades y características de las personas inmigrantes, sino que fomenten su participación activa y permitan desarrollar un posterior proceso de empoderamiento. Sería conveniente a su vez, resaltar la importancia de la participación de las familias y del grueso de la comunidad en estos procesos de inclusión. En este sentido querríamos concluir indicando que ha de posibilitarse la confluencia de diferentes iniciativas provenientes de la administración, de movimientos de TLE, de entidades de apoyo a inmigrantes, de entidades comunitarias o de las propias comunidades de inmigrantes; trabajando en red, compartiendo conocimientos y capacidades para poder así posibilitar una sociedad inclusiva. Este sería en definitiva nuestro principal el reto, y el tiempo libre educativo jugaría un papel profundamente relevante para poder avanzar en esa dirección. 
AINSCROW, M. (2004): Desarrollo de escuelas inclusivas. Ideas, propuestas y experiencias para mejorar las instituciones escolares, Narcea, Madrid.

ARMENGOL, C. (2012): “La investigación en el tiempo libre de niños y jóvenes: panorámica de la diversidad", Revista de intervención socioeducativa, 50, 45-67.

AYARZA, E.; FERNANDEZ, A. M. y MENDIA, R. (2010): Aproximacion al enfoque educativo del tiempo libre en el siglo XXI, Bilbao, Fundacion Aisi-Hezi.

BERRY, J. (1997): “Inmigration, Acculturation and Adaptation”, Applied Psychology, 46, 5-68.

BERRY, J.W.; PHINNEY, J.S.; SAM, D. L. y VEDDER, P. (2006): "Immigrant youth: Acculturation, identity, and adaptation”, Applied psychology, 55(3), 303332.

DALI, K. (2012): “Reading their way through immigration: The leisure reading practices of Russianspeaking immigrants in Canada", Library of Information Science Research, 34, 197-211.

DEVINE, M.A. y DATTILO, J. (2000): "Social acceptance and leisure lifestyles of people with disabilities", Therapeutic Recreation Journal, 34(4), 306-322.

FANTOVA, F. (2004): Ocio, inclusión y discapacidad. Un marco para el desarrollo y la mejora de servicios de ocio inclusivo, Universidad de Deusto, Bilbao. Recuperado de [<http://www. deusto-publicaciones.es/deusto/pdfs/ocio/ ocio28.pdf〉].

FERTIG, M. (2010): “The Societal Integration of Immigrants in Germany", en Epstein, G. y Gang, I. (ed.) Migration and Culture (Frontiers of Economics and Globalization, volumen 8) Emerald Group Publishing Limited, 375-400.
FLOOD, J.P. y McAVOY, L.H. (2007): "Use of national forests by Salish-Kootenai tribal members: Traditional recreation and a legacy of cultural values", Leisure/Loisir, 31, 191-216.

GIBSON, J. (2001): "Making Tolerance Judgements: The Effect of Context, Local and National", The Journal of Politics, 63, 1.067-1.090.

GONZÁLEZ-LÓPEZ, J.R.; RODRÍGUEZ-GÁZQUEZ, M. y LOMASCAMPOS, M. (2015): "Physical Activity in Latin American Immigrant Adults Living in Seville, Spain”, Nursing research, 64(6), 476-484.

HIBBLER, D.K. y SHINEW, K.J. (2002): "The Role of Leisure Service Providers in Enhancing Multiracial Families' Leisure Experiences", Parks \& Recreation, 37(2), 26-33.

HÖLGAMMER, A.; STOKOWSKI, P.; MUHAR, A.; SCHAUPPENLEHNER, T.; YALCINTEPE, E. y RENNER, J. (2015): “Experiences and meanings of leisure for members of the Turkish and Chinese communities in Vienna, Austria", World Leisure Journal, 57(3), 196-208.

IKUSPEGI (2017): Evolución de la población total y de origen extranjero en la CAE y territorios históricos 1998-2017. Recuperado de [/http://www. ikuspegi.eus/documentos/tablas/castellano/ evolucion/2017/Evolucion_extranj_total_2017. pdf>].

INSTITUTO NACIONAL DE ESTADÍSTICA (2016): Estadística de padrón continuo a 1 de Enero de 2016. Recuperado de [rhttp://www.ine.es/prensa/ np966.pdf)].

KIM, J. E IWASAKI, Y. (2016): "Role of leisure-generated meanings in adaptation to acculturation stress of Korean immigrants in Canada", Loisir et Societé/Society and Leisure, 39:2, 177-194. 
KIM, J.; HEO, J. y LEE, C. (2015): "Exploring the relationship between types of leisure activities and acculturation among Korean immigrants", Leisure Studies. 35, 113-127.

LIU, J.; PROBST, J.C.; HARUN, N.; BENNETT, K.J. y TORRES, M.E. (2009): "Acculturation, physical activity, and obesity among Hispanic adolescents", Ethnicity and Health, 14, 509-525.

LIVENGOOD, J.S. y STODOLSKA, M. (2004): “The effects of discrimination and constraints negotiation on leisure behavior of American Muslims in the post-September 11 in America", Journal of Leisure Research, 36(2), 183.

MACNEIL, R.D. y ANDERSON, S.C. (1999): “Leisure and persons with developmental disabilities: Empowering self-determination through inclusion", en Retish, P. y Reiter, S. (eds.): Adults with disabilities: International perspectives in the community, 125-143, Lawrence Erlbaum, Mahwah.
MALMUSI, D. y JANSA I LOPEZ DEL VALLADO, J.M. (2007): "Recomendaciones para la investigación e información en salud sobre definiciones y variables para el estudio de la población inmigrante de origen extranjero", Revista Española de Salud Pública, 81(4), 399-409.

MORATA, T., (2015): Memoria científico-técnica de proyecto de investigación I+D+i; ocio, acción sociocultural y cohesión social (inédito).

SHARAIEVSKA, I.; STODOLSKA, M.; SHINEW, K. J. y KIM, J. (2010): "Perceived discrimination in leisure settings in Latino urban communities". Leisure/ Loisir, 34, 295-326.

STODOLSKA, M. (2015): “Recreation for all: Providing leisure and recreation services in multi-ethnic communities", World Leisure Journal, 57(2), 89-103.

STODOLSKA, M. y ALEXANDRIS, K. (2004): "The role of recreational sport in the adaptation of first generation immigrants in the United States", Journal of Leisure Research, 36(3), 379.

\section{Direcciones de interés sobre buenas prácticas}

Somos Rontegi Gara: [<http://www.barakaldo.org/portal/ web/accion-social/detalle/-/asset_publisher/ Pomq/content/somos-rontegi-gara〉]

Bizilagunak [khttp://www.barakaldo.org/portal/web/ accion-social/inmigracion/bizilagunak>]

Munduko Arrozak: [〈http://mundukoarrozak.info〉]

Giltzarri: [〈http://www.giltzarri.info〉]

Auzoko: [khttps://goiena.eus/bergara/1386609059064auzoko-egitasmoa-euskaldunak-eta-etorkinakelkarrekin-euskara-lantzen〉]
Proyecto antirrumores: [<http://www.bilbao.eus/cs/ Satellite? c=BIO_Noticia_FA\&cid $=12791682151$ 21\&language $=e s \&$ pageid $=3012593044 \&$ page name $=$ Bilbaonet\%2FBIO_Noticia_FA\%2FBIO Noticia>]

Fundación Harribide: [〈http://www.harribide.org〉]

Fundación Ellacuría Transpirenaica Social y Solidaria: [〈http://transpirenaicasocialsolidaria.org 〉]

Gauean: [<https://www.lailusionquenosmueve.com/ ilusiones-sin-limites/gauean-albergue-parajovenes-extranjeros-sin-techo/>] 